\title{
Erratum to: Acute response test to adaptive servo-ventilation, a possible modality to assessing the reversibility of pulmonary vascular resistance
}

\author{
Michinari Hieda $^{1} \cdot$ Osamu Seguchi $^{1} \cdot$ Yoshihiro Mutara $^{1} \cdot$ Haruki Sunami $^{1}$. \\ Takuma Sato $^{1} \cdot$ Masanobu Yanase $^{1} \cdot$ Hata Hiroki $^{2}$. Tomoyuki Fujita ${ }^{2} \cdot$ \\ Takeshi Nakatani ${ }^{1}$
}

Published online: 20 May 2015

(C) The Japanese Society for Artificial Organs 2015

\section{Erratum to: J Artif Organs \\ DOI 10.1007/s10047-015-0833-1}

In the original publication of the article, Table 1 was published incorrectly. The correct Table 1 should be as below:

Table 1 Hemodynamic measurements on first RHC, first RHC with 101 oxygen and ASV, second RHC and third RHC after LVAD implantation

\begin{tabular}{|c|c|c|c|c|c|}
\hline & \multicolumn{3}{|c|}{ First RHC } & \multirow{2}{*}{$\begin{array}{l}\text { Second RHC } \\
\text { (LVAD } 1 \text { month) }\end{array}$} & \multirow{2}{*}{$\begin{array}{l}\text { Third RHC } \\
\text { (LVAD } 5 \text { months) }\end{array}$} \\
\hline & & $\left(\begin{array}{llll}10 & 1 & O_{2}\end{array}\right)$ & $(\mathrm{ASV})$ & & \\
\hline PCWP (mmHg) & 23 & 28 & 29 & 7 & 10 \\
\hline PAP (mmHg) & $65 / 30 / 45$ & $52 / 28 / 36$ & $52 / 29 / 36$ & $38 / 19 / 26$ & $32 / 15 / 22$ \\
\hline $\mathrm{CO}(1 / \mathrm{min})$ & 3.71 & 3.49 & 3.25 & 3.85 & 5.00 \\
\hline CI $\left(1 / \mathrm{min} / \mathrm{m}^{2}\right)$ & 2.01 & 1.90 & 1.76 & 2.25 & 2.94 \\
\hline PVR (Wood units) & 5.93 & 2.29 & 2.15 & 4.92 & 2.40 \\
\hline
\end{tabular}

$R H C$ right heart catheterization, $L V A D$ left ventricular assisted device, $A S V$ adaptive servo-ventilation, $P C W P$ pulmonary capillary wedge pressure, $P A P$ pulmonary arterial pressure, $C O$ cardiac output, $C I$ cardiac index, $P V R$ pulmonary vascular resistance

The online version of the original article can be found under doi:10.1007/s10047-015-0833-1.

\section{Michinari Hieda}

hieda_michinari_0119@yahoo.co.jp

1 Department of Transplantation, National Cerebral and Cardiovascular Center, 5-7-1 Fujishiro-dai, Suita,

Osaka 565-8565, Japan

2 Department of Adult Cardiac Surgery, National Cerebral and Cardiovascular Center, Osaka, Japan 